\title{
Talla de captura y reproducción de la langosta verde Panulirus gracilis (Decapoda: Palinuridae) en la costa de Manabí, Ecuador
}

\author{
Juan Figueroa \& David Mero \\ Universidad Laica Eloy Alfaro de Manabí. Departamento Central de Investigación, Cdla. Universitaria Vía San \\ Mateo, Manta, Ecuador; alberto.figueroa@uleam.edu.ec, varulv969@hotmail.com, david.mero@uleam.edu.ec, \\ david-j@hotmail.es
}

Recibido 10-V-2012. Corregido 05-X-2012. Aceptado 30-X-2012.

\begin{abstract}
Length of capture and reproduction of green spiny lobster Panulirus gracilis (Decapoda: Palinuridae) from the coast of Manabí, Ecuador. Green spiny lobster Panulirus gracilis is an economical resource of high importance for fishermen populations in the continental coast of Ecuador. Traditionally, this specie is captured using gillnets and semiautonomous diving system (hookah). With the objective to analyze some biological aspects about this fishery in Manabí, we examined lengths of capture (carapace length: CL and abdominal length: AL), sex ratio and reproductive aspects in females between June and September 2010. A total of 415 females and 288 males were captured with gillnets in El Mangle-Puerto Cayo (Central coast, depth: 3-6m), and by diving in Puerto López-Salango (South coast, depth: 7-12m). Sex ratio from males to females was $0.72: 1$ for gillnets, and $0.63: 1$ for diving. Lobsters captured by diving were larger $(84.1 \pm 3.3 \mathrm{~mm} \mathrm{CL})$ than lobsters captured by gillnets $(73.4 \pm 2.9 \mathrm{~mm} \mathrm{CL})$. Percentage of ovigerous females captured by diving was higher than females captured by gillnets. There was a pattern between lengths by sex (relation AL vs. CL); females were larger than males at same CL. Differences found between lengths and the occurrence of ovigerous females could be related with depth where capture methods are used. A total of $98 \%$ of landed lobsters were smaller than legal captured length and we recommend the establishment of a monitoring evaluation program during the fishing season, and a ban, in order to evaluate the natural fluctuation in length and reproductive stages of green spiny lobster in the coasts of Manabí. Rev. Biol. Trop. 61 (3): 1189-1199. Epub 2013 September 01.
\end{abstract}

Key words: Panulirus gracilis, green spiny lobster, length of capture, reproductive aspects, gillnets, diving, Ecuador.

La langosta verde Panulirus gracilis (Streets 1871) es un recurso de alta importancia económica para las poblaciones pesqueras de la costa continental del Ecuador, debido a su alto valor adquisitivo (Villón et al. 2000). Un promedio de 145 toneladas anuales de $P$. gracilis y $P$. penicillatus (especie solo encontrada en Galápagos) son exportadas desde Ecuador hacia Italia, Estados Unidos, Bélgica y España (Fedexpor 2008) por lo que esta especie se considera como una importante fuente generadora de divisas para el país. La explotación del recurso langosta se extiende tradicionalmente a todas las provincias de la costa continental del Ecuador, concentrándose mayoritariamente en: Esmeraldas, Manabí, Santa Elena y Guayas (Villón et al. 2000).

$P$. gracilis es capturada en la costa continental del Ecuador desde 1960 (Loesch \& López 1966, Cun \& Campos 1993, Villón et al. 2000). Los métodos de captura de esta especie incluyen el uso tradicional de redes de fondo y a partir de la década de los ochenta el buceo semiautónomo (Correa et al. 1994). Las redes son colocadas en fondos rocosos o gravosos poco profundos y con elevada turbidez, donde la langosta verde se agrega (Briones \& Lozano 2000). Este método pasivo tiene una alta efectividad de captura pudiendo atrapar individuos juveniles y hembras ovígeras. El método de 
captura con buceo semiautónomo (hookah) se extendió rápidamente en la costa continental del Ecuador como producto de la creciente tecnificación de los pescadores artesanales (Correa et al. 1994, Gaibor 2002). Con este método activo las langostas son capturadas mediante varillas de metal, trinches o ganchos sin diferenciación en las tallas, lo cual también incluye hembras ovígeras.

La creciente demanda por este recurso originó el aumento del esfuerzo pesquero (número de pescadores, embarcaciones y días de pesca). A inicios de los años ochenta empezó a registrarse un aumento en la captura de langostas con tallas menores a $260 \mathrm{~mm}$ de longitud total (LT) y hembras ovígeras en los desembarques (Cun \& Campos 1993, Villón et al. 2000). El descenso en la talla media de captura ha sido documentada en la costa continental del Ecuador con 340mm LT en 1966 (Loesch \& López 1966); 242mm LT en 1979 (Cun \& Campos 1993); 195mm LT en 1994 (Correa et al. 1994); y 160mm LT en 2006 (Delhaye \& Ormaza 2006). Para el 2011, esta tendencia de disminución en las tallas de las langostas se ha mantenido, indistintamente del método de captura empleado, lo cual sugiere un manejo inadecuado del recurso.

En 1993 el gobierno ecuatoriano decretó una veda total de siete años para evitar la captura de langostas con tallas menores a la legal (260mm LT) y hembras ovígeras (Registro Oficial 075, MICIP/SRP, 8 de julio de 1993), para de esta manera permitir: la repoblación natural de esta pesquería y la racionalización de su explotación (Campos 1993, Correa et al. 1994, Villón et al. 2000). Sin embargo, esta decisión no fue efectiva, pues no estuvo acompañada de un programa de control-vigilancia de la pesquería ilegal, monitoreo y evaluación del recurso. Desde su reapertura en el 2000, se decretó un período de veda cada año entre el 16 enero y el 16 junio (Acuerdo Ministerial 182, MICIP/SRP, 27 noviembre 2001), bajo la consideración de colectar información biológica pesquera que permitiera contar con argumentos técnicos que respaldaran la aplicación de políticas de manejo sustentable del recurso.
No obstante, desde noviembre del año 2000, el Instituto Nacional de Pesca del Ecuador no ha publicado informes técnicos sobre la especie con la correspondiente inexistencia de información sobre su ciclo reproductivo anual, estado y fluctuación de sus poblaciones. Estos antecedentes muestran que la pesquería de la langosta verde $P$. gracilis en la costa continental del Ecuador presenta evidencias de una posible sobre-explotación debida a las incontroladas prácticas de pesca y a un manejo inadecuado. El propósito de este estudio fue analizar los aspectos biológicos y reproductivos de la langosta verde $P$. gracilis en relación con los métodos de captura utilizados en la costa de la provincia de Manabí, Ecuador.

\section{MATERIALES Y MÉTODOS}

Se analizó la información biológica y reproductiva de 703 langostas $P$. gracilis capturadas en dos zonas de la franja costera de Manabí y comercializadas en el desembarcadero y centro de acopio "Playita Mía", Manta, Manabí, Ecuador (0057’01"60" S $80^{\circ} 42^{\prime} 32$ ' 00 ” W), entre junio y septiembre del 2010. Las langostas de la zona centro-sur (El Mangle-Puerto Cayo, $\mathrm{n}=508$ ), fueron capturadas con red entre los 3-5m de profundidad; mientras las de la zona sur (Puerto LópezSalango, $\mathrm{n}=195$ ) fueron capturadas con buceo semiautónomo (hookah) entre los 7 y $12 \mathrm{~m}$ de profundidad.

La longitud cefalotoráxica (LC) de los organismos se midió desde la escotadura entre los cuernos supraorbitales hasta el extremo posterior del cefalotórax, y la longitud abdominal (LA) se midió desde el inicio del abdomen hasta el extremo posterior del telson. Las mediciones fueron realizadas con un calibrador vernier (precisión $\pm 0.01 \mathrm{~mm}$ ) y expresadas en milímetros (mm). El sexo de los organismos fue determinado mediante sus características macroscópicas externas, y se registró los estadios reproductivos de las hembras con base en la escala propuesta por Briones \& Lozano (1981). Para comparar la proporción de hembras entre métodos de captura, los especímenes 
fueron agrupados según su condición reproductiva en: 1) hembras no ovígeras (estadios I, II y VI) y 2) hembras ovígeras (estadios III, IV y V).

Los datos, proporción sexual, tallas y estadios reproductivos fueron analizados mediante histogramas de distribución de frecuencias. Para determinar diferencias en la proporción de sexos se utilizó la prueba Chi-cuadrado $\left(\chi^{2}\right)$ (Zar 1999):

$$
\chi^{2}=\Sigma\left[\left(f_{1}-f_{2}\right)-1\right]^{2} / n
$$

Donde $\mathrm{f}_{1}$ y $\mathrm{f}_{2}$ son número de machos y hembras, respectivamente (o de hembras y machos, según el sexo que sea más abundante) y n es el tamaño de la muestra total.

La prueba de análisis de varianza de dos vías se realizó para determinar las posibles diferencias e interacciones entre la talla (LC) por método de captura y sexo. La prueba de Tukey fue utilizada para determinar diferencias entre pares de medias con muestras de tamaño diferente. Se realizó un análisis de regresión lineal simple entre LA y LC para comparar patrones diferenciales de la talla por sexo. La diferencia entre pendientes se comparó mediante la prueba de homogeneidad de regresiones lineales (Zar 1999). El modelo de regresión lineal (LA y LC por sexo) fue ajustado mediante el método de mínimos cuadrados. Los datos fueron expresados como error estándar $( \pm 1 \mathrm{EE})$.
La frecuencia de aparición de hembras no ovígeras (estadios I, II, VI) y de hembras ovígeras (estadios III, IV y V) fue analizada utilizando tablas de contingencia y el estadístico $\chi^{2}$ con la prueba de corrección de Yates (Sokal \& Rohlf 1981).

\section{RESULTADOS}

Captura y proporción de sexos: El número de hembras fue mayor que el número de machos en ambos métodos de captura entre junio y septiembre de 2010 (Fig. 1), a excepción de agosto donde hubo un incremento de machos en la captura con red (Fig. 1). Hubo diferencias significativas en la proporción de sexos por método de captura. Para la captura con red, la proporción fue $0.72 \mathrm{M}: \mathrm{H}\left(\chi^{2}=12.92\right.$, g.l. $=1, \mathrm{p}=0.003)$ mientras que para la de buceo fue $0.63 \mathrm{M}: \mathrm{H}\left(\chi^{2}=19.93\right.$, g.l. $\left.=1, \mathrm{p}<0.001\right)$.

Capturas por talla y sexo: Las langostas capturadas con red y buceo tuvieron una talla promedio de $73.4 \pm 2.9 \mathrm{~mm}$ LC $(115.0-42.6 \mathrm{~mm}$ LC) y de $84.1 \pm 3.3 \mathrm{~mm} \mathrm{LC} \mathrm{(121.0-50.8mm} \mathrm{LC),}$ respectivamente (Fig. 2). Hubo diferencia significativa en la talla de las langostas por método de captura (Tukey, $\mathrm{p}=0.037$; Cuadro 1), pero no hubo diferencias por sexo (Tukey, $\mathrm{p}=0.506$; Cuadro 1). No hubo interacción entre los factores método de captura y sexo $(p=0.671$; Cuadro 1). Las langostas capturadas con buceo fueron más grandes que las capturadas con red.

CUADRO 1

ANOVA de dos vías (SC tipo I) de talla por método de captura y sexo de langostas Panulirus gracilis en Manabí, Ecuador. g.l. representa grados de libertad

TABLE 1

Two ways ANOVA (SS type I) for length in lobster Panulirus gracilis by method of capture and sex in Manabí, Ecuador. g.l. means freedom degrees

\begin{tabular}{lccccc}
\multicolumn{1}{c}{ Fuente de Variación } & SC & g.l. & CM & F & p \\
Método de captura & 390.28 & 1 & 390.28 & 5.81 & 0.0366 \\
Sexo & 31.86 & 1 & 31.86 & 0.47 & 0.5065 \\
Método de captura*Sexo & 12.83 & 1 & 12.83 & 0.19 & 0.6713 \\
Error & 671.33 & 10 & 67.13 & & \\
\hline
\end{tabular}




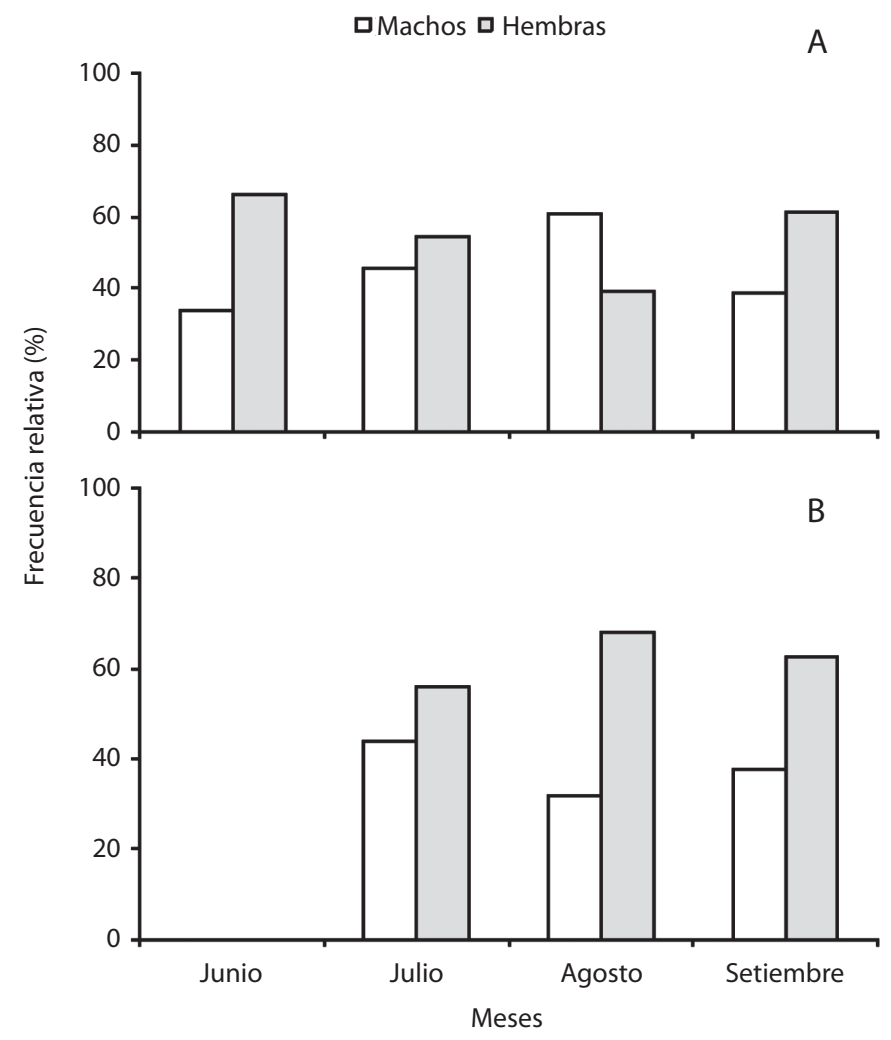

Fig. 1. Frecuencia relativa (\%) mensual de la proporción de sexos de Panulirus gracilis por método de captura en Manabí, Ecuador entre junio-septiembre 2010. (A) red y (B) buceo. En junio 2010 no hubo datos para buceo.

Fig. 1. Monthly relative frecuency for sex ratio of Panulirus gracilis by method of capture in Manabí, Ecuador between June-September 2010 (A) gillnets and (B) diving. No data for diving in June 2010.

El 98\% de las langostas capturadas se encontraron por debajo de la talla legal (103mm LC).

Relación morfométrica: El $\mathrm{r}^{2}$ fue 0.94 y 0.95 para machos y hembras respectivamente. Las ecuaciones obtenidas fueron $\mathrm{LA}=1.3936 \mathrm{LC}+7.2394$ (para machos) y $\mathrm{LA}=$ 1.7132LC-7.734 (para hembras). El modelo de regresión lineal (LA vs. LC) mostró divergencia entre las líneas de regresión por sexo, y un patrón alométrico diferencial entre las tallas de machos y hembras de P. gracilis (Fig. 3). Individuos machos de $80.0 \mathrm{~mm} \mathrm{LC}$, tuvieron $118.7 \mathrm{~mm}$ de LA $( \pm 0.77 \mathrm{~mm}$, intervalo de confianza al 95\%) mientras que las hembras tuvieron $129.3 \mathrm{~mm}$ de LA $( \pm 0.74 \mathrm{~mm}$, intervalo de confianza al 95\%). Las pendientes de regresión mostraron diferencias significativas $(\mathrm{p}=0.001)$.

Aspectos reproductivos: El 75\% y el $54 \%$ de las hembras capturadas con red y buceo, respectivamente, estuvieron en estadios reproductivos I y II (Fig. 4). Las proporciones de captura de los estadios III, IV y V, fueron altas en buceo con $15 \%, 17 \%$ y $10 \%$, respectivamente (Fig. 4). Con base a los resultados por corrección de Yates, hubo más hembras ovígeras en las capturas con buceo (71.4\%), que con $\operatorname{red}(25.5 \%)\left(\chi^{2}=19.43, p=0.05\right)$. Hubo un incremento en la proporción de hembras ovígeras en los meses de agosto y septiembre para ambos métodos de captura (Cuadro 2). 


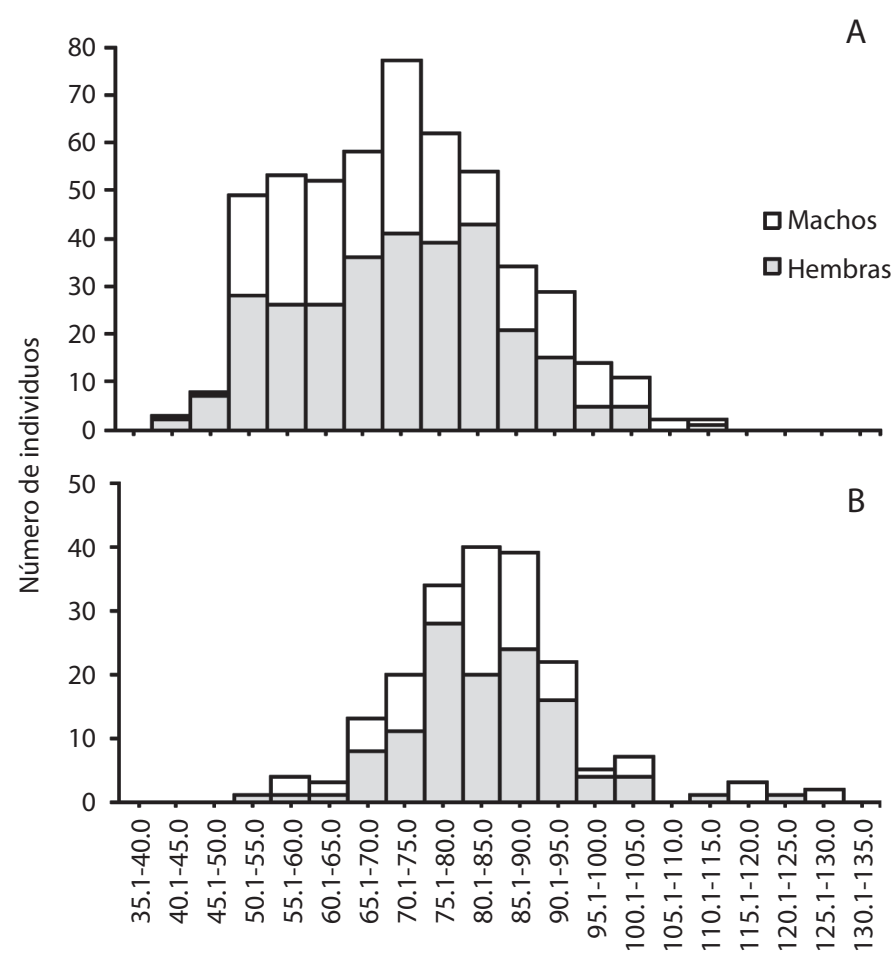

Longitud cefalotorácica $(\mathrm{mm})$

Fig. 2. Distribución de tallas de Panulirus gracilis por método de captura en Manabí, Ecuador entre junio-septiembre 2010 (A) red ( $\mathrm{n}=508)$ y (B) buceo $(\mathrm{n}=195)$.

Fig. 2. Size distribution of Panulirus gracilis by method of capture in Manabí, Ecuador between June-September 2010 (A) gillnets $(\mathrm{n}=508)$ and $(\mathbf{B})$ diving $(\mathrm{n}=195)$.

\section{DISCUSIÓN}

En este estudio se determinó que la proporción de machos fue menor que la proporción de hembras para ambos métodos de captura (red: 0.72:1 y buceo: $0.63: 1$ ). No obstante, varios autores señalan que en las capturas con red los machos de $P$. gracilis pueden llegar a ser más abundantes que las hembras. Loesch \& López (1966) y Cun \& Campos (1993) en estudios realizados en Ecuador reportan una proporción 1.5:1 y 2:1 respectivamente, mientras Briones \& Lozano (2003) en México reportan una proporción de 3.6:1 y López \& Sánchez (2010) en El Salvador de 2.3:1. Otros autores en Ecuador, México y Costa Rica reportan una proporción

\section{CUADRO 2}

Proporción mensual de hembras Panulirus gracilis no ovígeras (N.O.) y ovígeras (O.) por método de captura en Manabí, Ecuador. No hubo datos de captura con buceo junio 2010

\section{TABLE 2}

Monthly percentage of no ovigerous (N.O.) and ovigerous (O.) females of Panulirus gracilis by method of capture in Manabí, Ecuador. No data for diving in June 2010

\begin{tabular}{lcccc}
\multicolumn{1}{c}{ Mes } & \multicolumn{3}{c}{$\%$} \\
& Red & \multicolumn{2}{c}{ Buceo } \\
Junio & 0.98 & 0.02 & - & - \\
Julio & 0.80 & 0.20 & 0.64 & 0.36 \\
Agosto & 0.66 & 0.34 & 0.59 & 0.41 \\
Septiembre & 0.69 & 0.31 & 0.54 & 0.49 \\
\hline
\end{tabular}




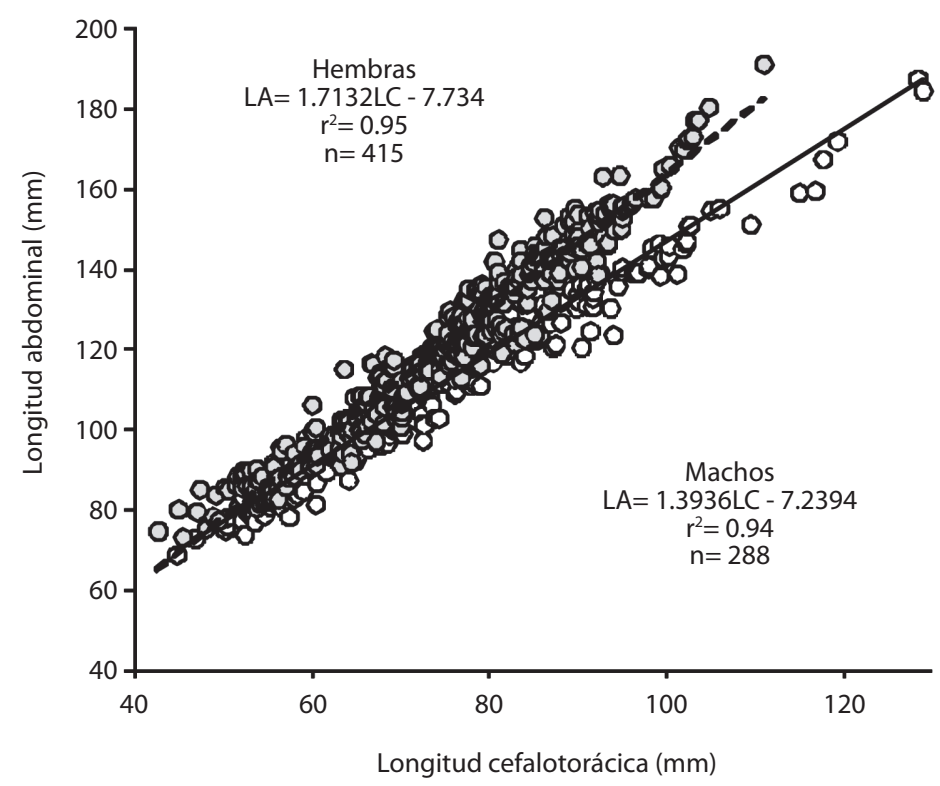

Fig. 3. Relación entre la longitud abdominal y la longitud cefalotorácica en machos y hembras de Panulirus gracilis en Manabí, Ecuador entre junio y septiembre 2010. Distancia entre rectas de regresión ( $\mathrm{p}<0.001)$.

Fig. 3. Relationship between abdominal length and carapace length for males and females of Panulirus gracilis in Manabí, Ecuador between June-September 2010. Distance between regression lines $(\mathrm{p}<0.001)$.

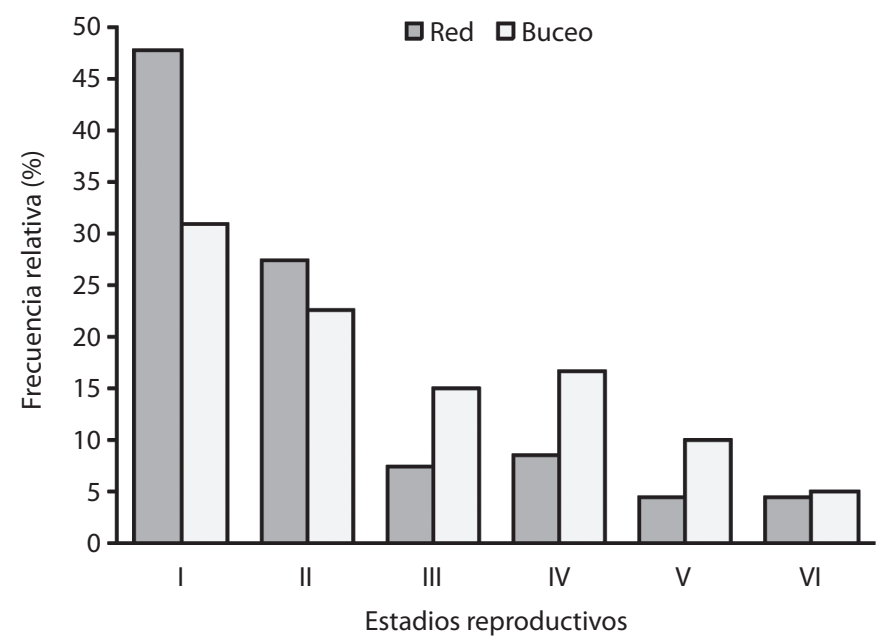

Fig. 4. Frecuencia relativa de estadios reproductivos (I-VI) en hembras Panulirus gracilis por método de captura en Manabí, Ecuador entre junio-septiembre 2010.

Fig. 4. Relative frecuency of reproductive stages (I-VI) in females of Panulirus gracilis by method of capture in Manabí, Ecuador between June-September 2010. 
cercana a la unidad (Briones \& Lozano 1981, Lozano et al. 1982, Villón et al. 2000, Patiño et al. 2009, Naranjo 2011). Krebs \& Davis (1993) sostienen que cuando la proporción de sexos es 1:1, el éxito reproductivo esperado de machos y hembras es igual y la población presenta características de estabilidad reproductiva.

Vega et al. (1992) mencionaron que las diferencias de las proporciones sexuales en las capturas de Panulirus interruptus (Randall 1842) se atribuyen al comportamiento reproductivo de las langostas en función al tipo de hábitat y por efecto de un gradiente latitudinal. Briones \& Lozano $(1992,2003)$ indican que las redes tienen mayor probabilidad de capturar machos que hembras ovígeras, porque las hembras cargadas con huevos tienen un comportamiento más solitario y permanecen más tiempo protegidas en sus refugios naturales. Por su parte, Naranjo (2011) indicó que las diferencias en las capturas por sexo pueden tener relación con la profundidad. Estrella \& McKiernan (1989) señalaron que las diferencias encontradas en la proporción de sexos de la langosta de que las Homarus americanus $(\mathrm{H}$. Milne-Edwards, 1837) en estratos profundos, podrían tener relación con el comportamiento reproductivo que tienen los machos adultos, que son territorialistas y compiten activamente por espacios de apareamiento.

Las langostas provenientes de Puerto López-Salango, capturadas mediante buceo fueron individuos adultos con talla promedio $84.1 \mathrm{~mm}$ de LC. Esta particularidad se debe a que los buzos pescadores de estas localidades seleccionan langostas de mayor tamaño, que permanecen ocultas en refugios de zonas distantes a la costa y con mejor visibilidad durante el día, entre 7 y $12 \mathrm{~m}$ de profundidad. Naranjo (2011) reporta para esta misma especie y el mismo método de captura, una talla media de $72.9 \mathrm{~mm}$ de LC a profundidades entre 11 y $35 \mathrm{~m}$.

Las langostas provenientes de El ManglePuerto Cayo capturadas con redes fueron individuos juveniles o preadultos con una talla promedio $73.4 \mathrm{~mm}$ LC. Esta talla de captura se debe a que los pescadores colocan sus redes en zonas cercanas a la costa con profundidades entre 3-5m durante la noche. Los individuos juveniles y preadultos prefieren ambientes poco profundos con alta turbidez, por lo que tienen mayor probabilidad de ser capturados en horas de la noche, cuando son más activos y realizan movimientos migratorios hacia zonas más profundas. López \& Sánchez (2010) realizaron un estudio en un complejo arrecifal de El Salvador y determinaron que las langostas capturadas con red, en estratos de profundidad de $2-5 \mathrm{~m}$, presentan una talla media $64 \mathrm{~mm}$ de LC.

Por lo expuesto, las diferencias en las proporciones sexuales y el incremento en la talla de captura de $P$. gracilis podría estar relacionado con la profundidad y la distancia de la costa, en los que los métodos de captura descritos son utilizados. Skud (1969) sugiere condiciones similares para estudios sobre Homarus americanus, remarcando que el incremento en el número de hembras sobre machos, $\mathrm{y}$ respecto al tamaño, es evidente en poblaciones de langostas adultas en ambientes profundos alejados de la costa, a diferencia de las poblaciones de juveniles que habitan en zonas menos profundas y cercanas a la costa.

En este estudio se observó un patrón diferencial de la talla por sexo en $P$. gracilis, siendo las hembras más grandes que los machos a una misma LC. Esto coindice con lo expuesto por De Barany et al. (1972), quienes sugirieron condiciones similares para Рапиlirus spp. en el Atlántico. Loesch \& López (1966) consideraron que éste patrón alométrico de talla por sexo empieza a hacerse evidente cuando las langostas alcanzan una talla de $220 \mathrm{~mm}$ de LT, lo cual coincidió con la primera talla de madurez sexual de las hembras en dicho estudio. Por su parte Guzmán et al. (2008) sostienen que este patrón alométrico entre sexos, es más evidente en adultos que en preadultos y juveniles; sin embargo, en nuestro estudio este patrón se presenta en hembras de aproximadamente 50mm LC $(\approx 128 \mathrm{~mm} \mathrm{LT})$. Según Briones \& Lozano. (1981, 1992, 2003), las hembras de $P$. gracilis alcanzan madurez sexual entre los 45 y $50 \mathrm{~mm}$ LC $(\approx 114-128 \mathrm{~mm}$ LT). Por lo tanto, el dimorfismo sexual respecto a la talla en $P$. gracilis podría ser efecto del 
primer evento reproductivo en las hembras, que empieza cuando los organismos alcanzan aproximadamente $50 \mathrm{~mm}$ LC, y podría irse acentuando conforme la hembra crece y se reproduce.

Nuestros resultados coinciden con los de Villón et al. (2000) quienes indican que el 98\% de las langostas capturadas en Ecuador continental están por debajo de la talla legal permitida (103mm LC). Esto podría explicar los cambios producidos en la estructura de tallas de la población que han venido ocurriendo por más de una década y que son evidentes en las distintas localidades pesqueras de Manabí, donde durante el período de pesca y también de forma ilegal durante el período de veda, se comercializan individuos juveniles y hembras ovígeras. Naranjo (2011) reportó que el $75 \%$ de organismos capturados en Guanacaste, Costa Rica estuvieron por debajo de la talla legal $(80 \mathrm{~mm} \mathrm{LC})$, lo que indica que la captura ilegal en las tallas de la langosta verde es un problema regional.

En este estudio el 27\% del total de hembras capturadas fueron ovígeras. En las capturas con red se encontraron hembras ovígeras con talla mínima $47 \mathrm{~mm}$ de LC ( $\approx 120 \mathrm{~mm} \mathrm{LT})$ mientras que en las capturas con buceo la talla mínima fue $69.2 \mathrm{~mm}$ de LC ( $\approx 180 \mathrm{~mm}$ LT). Velásquez \& Gutiérrez (2006), López \& Sánchez (2010) reportan hembras ovígeras para capturas con red a partir de, $65 \mathrm{~mm}$ y $52 \mathrm{~mm}$ de LC respectivamente, mientras Naranjo (2011) reporta hembras ovígeras en capturas con buceo con talla mínima 35.8mm LC. Estos estudios evidencian que $P$. gracilis puede empezar a madurar sexualmente a tallas pequeñas y posiblemente a edades relativamente tempranas. Cun \& Campos (1993) detectaron la presencia de hembras ovígeras de $170 \mathrm{~mm}$ LT y sostienen que esta particularidad podría atribuirse a factores ambientales (e.g. temperatura) que condicionan a las langostas a una actividad sexual precoz. Rothschild (1986) sugiere que la reducción en la densidad de los organismos de una población, puede resultar en el aumento de recursos alimenticios disponibles para los individuos, con el consecuente incremento en la tasa de desarrollo sexual y por tanto en el inicio de la madurez sexual a tamaños más pequeños. Por lo expuesto, la talla cada vez más pequeña de los organismos y el hecho que las hembras se estén reproduciendo bajo esa condición, podría indicar un desequilibrio en los procesos reproductivos y una estrategia de la especie como respuesta a la disminución paulatina en la densidad de organismos por presión pesquera y a una posible reducción en el número de hembras grandes (Polovina 1989).

Bohnsack et al. (2000) sugieren que las poblaciones pesqueras estables y sin riesgo de colapso, deben tener un porcentaje de potencial reproductivo del $50 \%$ con un valor crítico del $30 \%$. Por consiguiente, si el potencial reproductivo es menor al $30 \%$ no se permitiría un margen de confianza para la recuperación de las poblaciones que están sobreexplotadas. Velásquez et al. (2010) determinaron que las hembras de Panulirus inflatus con tallas menores a $80 \mathrm{~mm}$ LC poseen un potencial reproductivo menor al $11 \%$ y desovan hasta dos veces en un año, mientras que las hembras con tallas entre $80-110 \mathrm{~mm}$ de LC cuyo potencial reproductivo es superior al $80 \%$ pueden tener hasta cuatro desoves en un año. Figueroa (2012) determinó que las hembras de $P$. gracilis con LC entre 80 y $90 \mathrm{~mm}$, pueden producir un promedio de 400000 huevos por desove y que la fecundidad de las hembras en esta clase de talla puede superar hasta en un $80 \%$ a la fecundidad de hembras con tallas menores. Por lo expuesto, las poblaciones de langosta espinosa caracterizadas por una mayor abundancia de hembras ovígeras pequeñas y baja producción de huevos, podrían tener una capacidad mínima de recuperación frente a tasas de pesca altas y continuas. Esta hipótesis se apoya en lo encontrado por Berkeley et al. (2004) quienes sugieren la importancia de proteger a las hembras longevas, que a largo plazo pueden tener un rol fundamental en los procesos de reposición y estabilidad de las poblaciones pesqueras explotadas.

El incremento en la proporción de hembras ovígeras encontrado en los meses de agosto y septiembre para ambos métodos de 
captura coincide con lo reportado por el Instituto Nacional de Pesca (citado por Weinborn 1975 y Cun \& Campos 1993) hace más de 40 años. La temporada de pesca en la costa continental del Ecuador coincide con este pico reproductivo, lo cual dificulta cualquier estrategia de recuperación de las poblaciones de langosta. Sin embargo, sigue sin analizarse el ciclo reproductivo anual de $P$. gracilis, por lo que esta información es importante para establecer los meses en los que existe una mayor proporción de hembras ovígeras en las capturas, y promover su regulación.

De acuerdo con nuestros resultados, es posible que las hembras ovígeras sean más vulnerables a la captura con buceo, porque la reproducción se lleva a cabo en las zonas profundas y no en las zonas someras que es donde las redes son colocadas. Briones \& Lozano (1992) sostienen que la vulnerabilidad en la captura de hembras de $P$. gracilis con buceo podría atribuirse a que el hábitat de esta especie es poco intrincado, condición que les hace fácilmente detectable a la acción de los buzos. Esta condición es distinta en bajos rocosos de Manabí (buena visibilidad y profundidad mayor a $5 \mathrm{~m}$ ), donde la langosta verde $P$. gracilis se agrega a refugios naturales tipo grieta: intrincados, anchos y profundos (4-8 individuos/refugio) y a viseras del fondo poco intrincadas, estrechas poco profundas (1-2 individuos/refugio). Esta particularidad podría influir en la actividad de los buzos y por ende en la capturabilidad de las hembras ovígeras que debido a su comportamiento solitario podrían preferir las viseras (J. Figueroa obs. Pers. 2012).

Los resultados de talla de captura y aspectos reproductivos del presente estudio muestran que el recurso langosta verde en Manabí se encuentra en un posible proceso de sobre explotación, por lo cual se deben considerar futuras alternativas de evaluación del recurso, que permitan determinar el ciclo reproductivo y la variabilidad de la talla de captura de la especie durante todo el año. Esto constituye un aporte y sustento al establecimiento de las temporadas de pesca y veda con base científica, esperando un impacto a corto y mediano plazo con el consecuente beneficio para el sector pesquero.

Una estrategia de evaluación de las poblaciones de $P$. gracilis tendrá como resultado el manejo adecuado de la pesquería, considerando la articulación de acciones conjuntas entre el sector pesquero artesanal, científico y organismos de control. De esto se deriva, el posible establecimiento de una nueva talla legal de captura acorde con el estado actual de la población. Finalmente, uno de los factores de mayor importancia es la concienciación del pescador artesanal sobre el riesgo que conlleva la captura ilegal de organismos, lo cual influye negativamente en su economía y en los ecosistemas marinos.

\section{AGRADECIMIENTOS}

A Enrique Lozano, José Rivera, Patricia Briones, Jorge Sonnenholzner, Marcos Calle y Ricardo Castillo por la revisión y sugerencias hechas al manuscrito. A Carlos Bermúdez, Carlos Anchundia, Jhonny Delgado, Carolina Sierra, Pablo Zambrano, Christian Mantuano y Fabricio Ormaza por su colaboración. Este estudio fue financiado por la Subsecretaría de Recursos Pesqueros del Ministerio de Agricultura, Ganadería y Pesca del Ecuador (MAGAP/ SRP) y la Universidad Laica Eloy Alfaro de Manabí.

\section{RESUMEN}

La langosta verde Panulirus gracilis es un recurso de gran importancia económica para las poblaciones pesqueras de la costa continental del Ecuador. Esta especie es capturada tradicionalmente mediante redes y buceo semiautónomo (hookah). Para analizar algunos aspectos biológicos de la pesquería en la provincia de Manabí, se examinó la talla de captura (longitud cefalotorácica: LC y abdominal: LA), proporción sexual y aspectos reproductivos en hembras $(n=415)$ de 703 especímenes entre junio y septiembre 2010: con red en El Mangle-Puerto Cayo (costa central, profundidad: $3-6 \mathrm{~m}$ ), y mediante buceo en Puerto López-Salango (costa centro-sur, profundidad: 7-12m). La proporción sexual: machos a hembras fue 0.72:1 para captura con red y $0.63: 1$ para buceo. Las langostas capturadas con buceo fueron más grandes $(84.1 \pm 3.3 \mathrm{~mm} \mathrm{LC})$ que las capturadas con red $(73.4 \pm 2.9 \mathrm{~mm}$ LC). La proporción de 
hembras ovígeras capturadas con buceo fue mayor que en las capturas con red. La diferencia entre talla y ocurrencia de hembras ovígeras podría relacionarse con la profundidad a la que los métodos de captura fueron utilizados. El 98\% de las langostas analizadas estuvieron por debajo de la talla legal. Se propone el establecimiento de un programa de evaluación continua del recurso durante la temporada de pesca y veda para conocer la fluctuación natural del tamaño y época reproductiva de la langosta verde en Manabí.

Palabras clave: Panulirus gracilis, langosta verde, talla de captura, aspectos reproductivos, red, buceo, Ecuador.

\section{REFERENCIAS}

Berkeley, S.A., C. Chapman \& S.M. Sogard. 2004. Maternal age as a determinant of larval growthand survival in a marine fish, Sebastes melanops. Ecology 85: $1258-1264$

Bohnsack, J.A., B. Causey, M.P. Crosby, R.B. Griffis, M.A. Hixon, T.F. Hourigan, K.H. Koltes, J.E. Maragoss, A. Simons \& J.T. Tilmant. 2000. A rationale for minimum $20-30 \%$ no-take protection, p. 23-27. In Proceedings 9th International Coral Reef Symposium, Bali, Indonesia.

Briones, P. \& E. Lozano. 1981. Aspectos generales de la biología y pesca de las langostas en Zihuatanejo, Gro., México (Crustacea: Palinuridae). An. Inst. Cienc. Mar Limnol. Univ. Nac. Autón. México. 8: 79-102.

Briones, P. \& E. Lozano. 1992. Aspects of the reproduction of Panulirus inflatus (Bouvier) and Panulirus gracilis Streets (Decapoda: Palinudidae) from the Pacific Coast of Mexico. J. Crust. Biol. 12: 41-50.

Briones, P. \& E. Lozano. 2000. The spiny lobsters fisheries in México, p. 169-188. In B.F. Phillips \& J. Kittata (eds.). Spiny Lobsters: Fisheries and Culture. Fishing News Books, Oxford, Inglaterra.

Briones, P. \& E. Lozano. 2003. Factors affecting growth of the spiny lobsters Panulirus gracilis and Panulirus inflatus (Decapoda: Palinuridae) in Guerrero, México. Rev. Biol. Trop. 51: 165-174.

Campos, J. 1993. Informe sobre la situación actual de la langosta en la región insular y continental del Ecuador. Instituto Nacional de Pesca, Departamento de Ecología Marina, Ecuador.

Correa, J., M. Herrera, G. Villanueva \& J. Campos. 1994. Informe sobre el recurso langosta en la región insular y continental del Ecuador. Instituto Nacional de Pesca. Departamento de Ecología Marina, Ecuador.

Cun, M. \& J. Campos. 1993. Estudio de las capturas comerciales de Panulirus gracilis (Langosta verde) del noreste del golfo de Guayaquil (1970-1979). Bol.
Cient. Tec. Instituto Nacional de Pesca Ecuador 12: 1-19.

De Barany, T., J. Ewald \& E. Cadima. 1972. La pesca de la langosta en el Archipiélago de los Roques, Venezuela. I/T. Caracas. 43: 1-34.

Delhaye, L. \& C. Ormaza. 2006. Aspectos biométricos de las capturas de langosta espinosa Panulirus gracilis (Streets, 1871) en las costas de Jaramijó, Manabí, Ecuador. Tesis de Licenciatura, Universidad Laica Eloy Alfaro de Manabí, Ecuador.

Estrella, T. \& J. McKiernan. 1989. Catch-per-unit effort and biological parameters from Massachusetts Coastal Lobster (Homarus americanus) Resource: Description and Trends. NOAA Technical Report NMFS 81. NOAA/National Marine Fisheries Service, EE.UU.

Fedexpor 2008. Federación Ecuatoriana de Exportadores. Manta-Ecuador (Consultado: 7 julio 2012, http:// www.fedexpor.com).

Figueroa, J. 2012. Estudio del recurso pesquero langosta verde Panulirus gracilis en Manta y Jaramijó, Manabí, Ecuador. Informe final de Resultados. Departamento Central de Investigación. Universidad Laica Eloy Alfaro de Manabí, Manabí, Ecuador.

Gaibor, N., J. Rosero \& M. Altamirano. 2002. El impacto de la migración humana en las artes de pesca artesanales y semi industriales utilizadas en los parques nacionales Galápagos (Isla Isabela) y Machalilla. Informe Técnico de Consultoría para The Nature Conservancy y Fundación Natura, Guayaquil, Ecuador.

Guzmán, H.M., R. Cipriani, A.J. Vega, M. López \& J.M. Meir. 2008. Population Assessment of the Pacific Green Spiny Lobster (Panulirus gracilis) in the Pacific of Panama. J. Shellfish. Res. 43: 14-23.

Krebs, J. \& N. Davies. 1993. An introduction to behavioural ecology. Blackwell, Oxford, Inglaterra.

Loesch, H. \& E. López. 1966. Observaciones sobre la langosta de la costa del Ecuador. Bol. Cient. Tec. Instituto Nacional de Pesca, Ecuador 1: 1-30.

López, W. \& J. Sánchez. 2010. Pesca de Panulirus gracilis (Crustacea: Palinuridae) en el complejo recifal los Cóbanos, El Salvador XVI. Simposio Ibérico de Estudios de Biología Marina, Alicante, España.

Lozano, E., P. Briones, L. Santarelli \& A. Gracia. 1982. Densidad poblacional de Panulirus gracilis Streets y $P$. inflatus (Bouvier) (Crustacea: Palinuridae) en dos áreas cercanas a Zihuatanejo, Gro., México. CC. Pesq. 3: 61-73.

Naranjo, H. 2011. Biología pesquera de la langosta Panulirus gracilis en Playa Lagarto, Guanacaste, Costa Rica. Rev. Biol. Trop. 59: 619-633.

Patiño, J., J. Tovar, P. Ulloa, L. Guevara \& R. Sánchez. 2009. Descripción de la pesquería de langosta en Nayarit, México, durante el período 2001-2006. CC. Pesq. 17: 41-49. 
Polovina, J.J. 1989. Density dependence in spiny lobster, Panulirus marginatus, in the Northwestern Hawaiian Islands. Can. J. Fish. Aquat. Sci. 46: 660-665.

Rothschild, B.I. 1986. Dynamics of marine fish populations. Harvard University, Cambridge, Inglaterra.

Sokal, R. \& F. Rohlf. 1981. Biometría: Principios y Métodos Estadísticos en la Investigación Biológica. Blume, Madrid, España.

Skud, B. 1969. The effect of fishing on size composition and sex ratio of offshore lobster stocks. U.S. Bureau of Commercial Fisheries, Biological Laboratory, Boothbay Harbor, Maine. FiskDir. Skr. Ser. HavUnds. 15: 295-309.

Vega, A., G. Espinoza, C. Castro, G. León, J. Turrubiates \& M. Reineke. 1992. Variación espacio-temporal de la estructura poblacional e índices de abundancia relativa y su relación con el proceso de reclutamiento de las langostas (Panulirus spp.), en el litoral occidental de Baja California Sur, p. 45. In S. Guzmán del Pró (ed.). Memorias del Taller México-Australia sobre Reclutamiento de Recursos Bentónicos de Baja California, 25-29 noviembre 1991, La Paz, B.C.S. Secretaría de Pesca e Instituto Politécnico Nacional, México.
Velásquez, J.I., M. Villalejo \& A. Tripp. 2010. Fecundidad y proporción de sexos de Panulirus inflatus en la costa occidental del Golfo de California, México. Rev. Biol. Mar. Ocean. 45: 71-76.

Velásquez, L.E. \& R. Gutiérrez. 2006. Talla y peso de primera madurez sexual al $50 \%$ (Lm50 \%) y (Pm50 \%) para hembras de langosta verde Panulirus gracilis del Pacífico nicaragüense. Ministerio de Fomento, Industria y Comercio (MIFIC). Administración Nacional de Pesca y Acuicultura (ADPESCA). Centro de Investigaciones Pesqueras y Acuícolas CIPA/ ADPESCA, Nicaragua.

Villón, C., I. Cedeño, J. Correa \& M. Peralta. 2000. Situación actual del recurso langosta (Panulirus gracilis) en la costa continental ecuatoriana: Opciones de manejo para la pesquería, Instituto Nacional de Pesca, Ecuador.

Weinborn, J. 1975. Estudio preliminar de la biología, semicultivo y ecología de los Panuliridos de Zihuatanejo, Gro. Panulirus gracilis, Streets, 1871 y Panulirus inflatus, Bouvier 1895. UNAM, México.

Zar, J.H. 1999. Biostatistical Analysis. Prentice Hall, Upper Saddle River, Nueva Jersey, EE.UU. 
